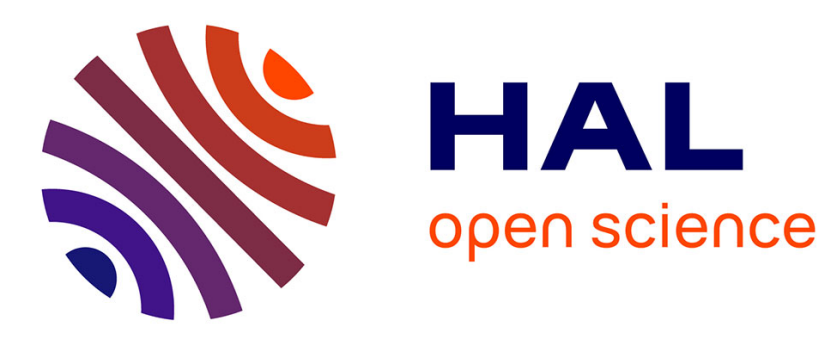

\title{
Symmetry-dependent field-free switching of perpendicular magnetization
}

Liang Liu, Chenghang Zhou, Xinyu Shu, Changjian Li, Tieyang Zhao, Weinan Lin, Jinyu Deng, Qidong Xie, Shaohai Chen, Jing Zhou, et al.

\section{- To cite this version:}

Liang Liu, Chenghang Zhou, Xinyu Shu, Changjian Li, Tieyang Zhao, et al.. Symmetry-dependent field-free switching of perpendicular magnetization. Nature Nanotechnology, 2021, 10.1038/s41565020-00826-8 . hal-03036308

\section{HAL Id: hal-03036308 https://hal.science/hal-03036308}

Submitted on 2 Dec 2020

HAL is a multi-disciplinary open access archive for the deposit and dissemination of scientific research documents, whether they are published or not. The documents may come from teaching and research institutions in France or abroad, or from public or private research centers.
L'archive ouverte pluridisciplinaire HAL, est destinée au dépôt et à la diffusion de documents scientifiques de niveau recherche, publiés ou non, émanant des établissements d'enseignement et de recherche français ou étrangers, des laboratoires publics ou privés. 
Symmetry-dependent field-free switching of perpendicular magnetization

Liang Liu ${ }^{1 \dagger}$, Chenghang Zhou ${ }^{1 \dagger}$, Xinyu Shu ${ }^{1}$, Changjian Li $^{1}$, Tieyang Zhao ${ }^{1}$, Weinan Lin ${ }^{1}$, Jinyu Deng ${ }^{1}$, Qidong Xie ${ }^{1}$, Shaohai Chen ${ }^{1}$, Jing Zhou ${ }^{1}$, Rui Guo ${ }^{1}$, Han Wang ${ }^{1}$, Jihang $\mathrm{Yu}^{1}$, Shu Shi ${ }^{1}$, Ping Yang ${ }^{1,2}$, Stephen Pennycook ${ }^{1}$, Aurelien Manchon ${ }^{3,4^{*}}$, Jingsheng Chen ${ }^{1 *}$

${ }^{1}$ Department of Materials Science and Engineering, National University of Singapore,

Singapore 117575

${ }^{2}$ Singapore Synchrotron Light Source (SSLS), National University of Singapore, 5 Research Link, Singapore 117603

${ }^{3}$ Physical Sciences and Engineering, King Abdullah University of Science and Technology, Thuwal 23955-6900, Saudi Arabia

${ }^{4}$ Aix-Marseille Univ, CNRS, CINaM, Marseille, France

${ }^{\dagger}$ These authors contributed equally to this work.

*e-mail:msecj@nus.edu.sg; manchon@cinam.univ-mrs.fr

Modern magnetic-memory technology requires all-electric control of perpendicular magnetization with low energy consumption. While spin-orbit torque (SOT) in heavy metal/ferromagnet $(\mathrm{HM} / \mathrm{FM})$ heterostructures ${ }^{1-5}$ holds promise for applications in magnetic random access memory, till today, it is limited to the in-plane direction. Such in-plane torque can switch perpendicular magnetization only deterministically with the help of additional symmetry breaking, e.g., through the application of an external magnetic field $^{2,4}$, an interlayer coupling ${ }^{6-9}$ or an asymmetric design ${ }^{10-14}$. Instead, an outof-plane spin-orbit torque ${ }^{15}$ could directly switch perpendicular magnetization. Here we 
observe an out-of-plane spin-orbit torque in an HM/FM bilayer of $L 1_{1}$-ordered $\mathrm{CuPt} / \mathrm{CoPt}$ and demonstrate field-free switching of the perpendicular magnetization of the CoPt layer. The low symmetry point group (3m1) at the $\mathrm{CuPt} / \mathrm{CoPt}$ interface gives rise to this spin torque, herein after referred as $3 \mathrm{~m}$ torque, which strongly depends on the relative orientation of current flow and crystal symmetry. We observe a 3-fold angular dependence in both the field-free switching and the current-induced out-ofplane effective field. Because of the intrinsic nature of the $3 \mathrm{~m}$ torque, the field-free switching in CuPt/CoPt shows good endurance in cycling experiments. Experiments with the wide variety of SOT bilayers with low-symmetry point groups ${ }^{16,17}$ at the interface may uncover further unconventional spin-torques in future.

According to the spin Hall scenario in an HM/FM bilayer, a charge current flowing in the HM layer with in-plane direction $(\boldsymbol{x})$ generates a spin current in the out-of-plane direction $(\boldsymbol{z})$ with spin polarization along the $y$-direction. When flowing into the FM layer, the spin current generates a damping-like torque whose direction is set by the spin-polarization direction $(y)$. This in-plane damping-like torque cannot deterministically switch the perpendicular magnetization. So far, two major solutions have been used to achieve the field-free perpendicular magnetization switching. One is by using the in-plane damping-like torque and a method to break the torque-symmetry, which includes engineering the exchange interaction $^{7-9} /$ interlayer coupling $^{6}$ along $\boldsymbol{x}$-direction and tilting the magnetization ${ }^{11,12}$ along $\boldsymbol{y}$ direction. The other is by generating an out-of-plane spin-torque along $z$-direction using an extra ferromagnetic layer ${ }^{15}$. However, all these designs complicate the device structure and may introduce extrinsic effects. Therefore, it is highly pursued to have an out-of-plane SOT in the HM/FM bilayer itself.

We deposited the $L 1_{1}$-ordered $\mathrm{CuPt}(10 \mathrm{~nm}) / \mathrm{CoPt}(4 \mathrm{~nm})$ bilayer on the $\mathrm{SrTiO}_{3}(111)$ substrate (see Methods). Compared with the interfacial magnetic anisotropy in conventional 
$\mathrm{HM} / \mathrm{FM}$ bilayers, the bulk magnetic anisotropy in $L 1_{1}$ CoPt enables higher memory density with an excellent thermal stability ${ }^{18,19}$. The crystal structure of $L 1_{1} \mathrm{CuPt}$ (or $L 1_{1} \mathrm{CoPt}$ ) is shown in Fig. 1a, where $\mathrm{Cu}(\mathrm{Co})$ and Pt atomic layers alternatively stack along the [111] direction in a rhombohedral structure. All crystallographic directions used in this study are denoted with the rhombohedral lattices. Fig. $1 \mathrm{~b}$ shows the plane view of the $L 1_{1} \mathrm{CuPt}$ crystal structure projected along the [111] direction, where there are three equivalent mirror planes [(1-10) and equivalent] and three planes without mirror symmetry [(11-2) and equivalent]. Therefore, in the (111) plane, we can define three high-symmetry axes ([11-2] and equivalent) and three low-symmetry axes ([1-10] and equivalent) (see Supplementary Fig. 1). The symmetry in Fig. $1 \mathrm{~b}$ is similar to that for $\mathrm{WTe}_{2}$, where the out-of-plane SOT was suggested to be allowed when the current is applied along the low-symmetry axis ${ }^{20}$. Supplementary Fig. 2 shows the $\theta-2 \theta$ X-ray diffraction spectra of our $\mathrm{CuPt} / \mathrm{CoPt}$ bilayer, where the (111) peak indicates the $L 1_{1}$ phase. The epitaxial growth of the bilayer was confirmed by the cross-sectional scanning transmission electron microscopy (STEM) in Fig. 1c, where we observed a sharp $\mathrm{CuPt} / \mathrm{CoPt}$ interface. The CuPt layer has a well-arranged atomically layered structure where the brighter atoms are $\mathrm{Pt}$ and the darker ones are $\mathrm{Cu}$ (see Supplementary Fig. $3 \mathrm{~b}$ for the schematic drawing). In contrast, for the CoPt layer, the intensity contrast of the elements is not sufficient for clearly distinguishing the Co and Pt atoms, which indicates a lower chemical ordering parameter in $\mathrm{CoPt}$, compared with that in $\mathrm{CuPt}^{21}$

The $L 1_{1} \mathrm{CuPt} / \mathrm{CoPt}$ bilayer was fabricated into $5 \mu \mathrm{m}$ Hall bar devices (Fig. 2a) with varying azimuth angles $\left(\theta_{I}\right)$ to the [1-10] direction (Fig. 2b). The anomalous Hall loops for $\theta_{I}$ $=0^{\circ}$ and other angles are shown in Fig. 2c and Supplementary Fig. 4, respectively. Supplementary Fig. 5 shows the magnetic hysteresis loops for the bilayer before patterning, which confirms the perpendicular magnetic anisotropy. Then we swept pulsed d.c. current 
(see Methods) and measured the Hall resistance change for Hall bars with different $\theta_{\mathrm{I}}$ (see Fig. 2e). No external magnetic field was applied for the measurement. With a current density of about $2.4 \times 10^{7} \mathrm{~A} / \mathrm{cm}^{2}$, the magnetization can be reversibly switched at angles $\theta_{\mathrm{I}}=0^{\circ}, 60^{\circ}$, $120^{\circ}$, and $180^{\circ}$. The switching polarity for $\theta_{\mathrm{I}}=0^{\circ}$ and $120^{\circ}$ is anti-clockwise while that for $\theta_{\mathrm{I}}$ $=60^{\circ}$ and $180^{\circ}$ is clockwise. For $\theta_{\mathrm{I}}=30^{\circ}, 90^{\circ}$, and $150^{\circ}$, the switching loop vanishes, which means the magnetization cannot be switched by the current. We define $\Delta R_{\mathrm{I}}$ as the difference in Hall resistance at zero current after current pulsing with $+20 \mathrm{~mA}$ and $-20 \mathrm{~mA}$, respectively. We found the $\theta_{\mathrm{I}}$ dependence of $\Delta R_{\mathrm{I}}$ shows a 3-fold symmetry (Fig. 2d). The field-free switching occurs when the current is along the low-symmetry axes, whereas it is prohibited when the current is along the high-symmetry axes. It is worth noting that the field-free switching (with smaller $\left.\Delta R_{\mathrm{I}}\right)$ also exists at some other values of $\theta_{\mathrm{I}}\left(15^{\circ}, 45^{\circ}, 75^{\circ}, 105^{\circ}, 135^{\circ}\right.$, $165^{\circ}$ ) that are neither along the high-symmetry axes nor along the low-symmetry axes (see Supplementary Fig. 6). We can use a cosine function to fit the $\theta_{\mathrm{I}}$ dependence of $\Delta R_{\mathrm{I}}$ with a period of $120^{\circ}$, as shown in Fig. 2 d. To understand this symmetry-dependent field-free switching, we measure the current-induced effective fields.

We first estimated the in-plane damping-like effective field of the $\mathrm{CuPt} / \mathrm{CoPt}$ bilayer by harmonic Hall voltage analysis ${ }^{22-25}$. Fig. 3a shows the first $\left(V_{\omega}\right)$ and second $\left(V_{2 \omega}\right)$ harmonic Hall signals for the Hall bar with $\theta_{I}=0^{\circ}$ in a small in-plane field $(H)$ range. The harmonic Hall signals for other $\theta_{I}$ are shown in Supplementary Fig. 7. After subtracting thermoelectric contribution (Supplementary Fig. 8), we obtain the damping-like effective field by using the formula $^{23}$

$$
\Delta H_{D L}=-2 \frac{B_{L}+2 \xi B_{T}}{1-4 \xi^{2}}
$$

where $B_{L(T)}$ is defined as $\left\{\frac{\partial V_{2 \omega}}{\partial H} / \frac{\partial^{2} V_{\omega}}{\partial H^{2}}\right\}_{L(T)} . \xi \sim 0.07$ is the ratio of planar Hall voltage to anomalous Hall voltage. The current dependence of $\Delta H_{\mathrm{DL}}$ is shown in Fig. $3 \mathrm{~b}$. The results for 
$0^{\circ}$ and $60^{\circ}$ (low-symmetry axes) are almost the same as that for 30 and $90^{\circ}$ (high-symmetry axes). According to the linear fit, the spin torque efficiency $\left(\mu_{0} \Delta H_{\mathrm{DL}} / J_{a c}\right)$ is calculated to be 9 $\mathrm{mT} /\left(1 \times 10^{8} \mathrm{~A} / \mathrm{cm}^{2}\right)$ and remains almost constant with a variation smaller than $1 \mathrm{mT} /\left(1 \times 10^{8}\right.$ $\mathrm{A} / \mathrm{cm}^{2}$ ) for varying $\theta_{I}$, as shown in Fig. 3c. In general, the in-plane damping-like torque in the HM/FM bilayer was considered to mainly originate from the spin Hall effect of the HM layer. Our result indicates an isotropic spin Hall angle, consistent with a previous report ${ }^{26}$. Then we characterized the out-of-plane SOT effective field $\left(\Delta H_{\mathrm{OOP}}\right)$ by measuring the anomalous Hall loop under applying different d.c. currents ${ }^{10,27}$. As shown in Fig. 3d, when we apply a positive $(+18 \mathrm{~mA})$ and a negative $(-18 \mathrm{~mA})$ pulsed currents $\left(30 \mu\right.$ s duration) into the Hall bar with $\theta_{I}=$ $0^{\circ}$, the centers of the anomalous Hall loop are shifted to the left and the right, respectively. Based on the loop shift, a $\Delta H_{\mathrm{OOP}}$ of $+0.8 \mathrm{mT}(-0.8 \mathrm{mT})$ is estimated for $+18 \mathrm{~mA}(-18 \mathrm{~mA})$. While for $\theta_{I}=60^{\circ}$, the loop shifts are opposite to that for $0^{\circ}$, which give a negative (positive) $\Delta H_{\mathrm{OOP}}$ for a positive (negative) current (see Fig. 3f). For $\theta_{I}=30^{\circ}$ (Fig. 3e) and $\theta_{I}=90^{\circ}$ (Fig. $3 \mathrm{~g}$ ), the loop shift vanishes, which gives a $\Delta H_{\mathrm{OOP}}$ of around 0 . Fig. $3 \mathrm{~h}$ shows the current dependence of $\Delta H_{\mathrm{OOP}}$ (see Supplementary Fig. 9-11). We found that $\Delta H_{\mathrm{OOP}}$ for $\theta_{I}=30^{\circ}$ and $\theta_{I}=90^{\circ}$ (high-symmetry axes) almost remains at 0 for all current values. In contrast, for the low-symmetry axis of $\theta_{I}=0^{\circ}\left(\theta_{I}=60^{\circ}\right), \Delta H_{\mathrm{OOP}}$ is close to 0 when the current is below 15 $\mathrm{mA}$, and then abruptly increases (decreases) with the current. We will discuss the possible origin of this threshold current observed in the $\mathrm{CuPt} / \mathrm{CoPt}$ bilayer further below. We estimated the out-of-plane spin-torque efficiency $\left(\mu_{0} \Delta H_{\mathrm{OOP}} / J_{\text {pulse }}\right)$ by using $\Delta H_{\mathrm{OOP}}$ measured at $\pm 18 \mathrm{~mA}$ for different $\theta_{I}$ (Supplementary Fig. 12). In Fig. 3i, $\mu_{0} \Delta H_{\mathrm{OOP}} / J_{\text {pulse }}$ shows a 3 -fold angular dependence on $\theta_{I}$, with an amplitude of $3.7 \mathrm{mT} /\left(1 \times 10^{8} \mathrm{~A} / \mathrm{cm}^{2}\right)$. The 3 -fold angular dependence of the out-of-plane effective field is consistent with the 3-fold field-free switching (Supplementary Fig. 13). In previous reports, the out-of-plane SOT effective field could be obtained after inducing an in-plane magnetization component $\left(\boldsymbol{M}_{\boldsymbol{x}}\right)$, through 
applying an in-plane magnetic field ${ }^{27}$ or by introducing an exchange bias ${ }^{8}$. These unidirectional controls should give a 1-fold angular dependent SOT switching. In contrast, the 3 -fold angular dependent field-free switching in $L 1_{1} \mathrm{CuPt} / \mathrm{CoPt}$ bilayer should have an intrinsic origin related to the crystal structure. As shown in Supplementary Fig. 14, after two thousand of repeated switching loops in a 26-days period, the switching behavior remains stable. We observed $\Delta R_{\mathrm{I}}$ almost keep unchanged (degradation $<4 \%$ ) with switching cycles, indicating an excellent switching performance. We also performed an endurance test for the $L 1_{1} \mathrm{CuPt} / \mathrm{CoPt}$ bilayer with a shorter pulse width (30 ns) and obtained an endurance up to $5 \times 10^{5}$ cycles (Supplementary Fig. 16).

The $L 1_{1}$ phase adopts the point group $R \overline{3} m$. Due to inversion symmetry breaking at the interface, the point group of the $\mathrm{CuPt} / \mathrm{CoPt}$ heterostructure reduces to $3 m 1$, i.e., a triad around [111] direction and a mirror plane normal to [1-10] direction. Applying these two symmetry operations to the current-driven spin-torque tensor, we obtain ${ }^{28}$

$$
\boldsymbol{T}=\chi_{D L} \boldsymbol{m} \times[(\mathbf{z} \times \boldsymbol{E}) \times \boldsymbol{m}]+\chi_{3 m} \boldsymbol{m} \times\left[\left(m_{y} E_{x}+m_{x} E_{y}\right) \boldsymbol{x}+\left(m_{x} E_{x}-m_{y} E_{y}\right) \boldsymbol{y}\right] .
$$

For short handedness, the Cartesian coordinates $(x, y, z)$ refer to the crystallographic directions ([1-10], [11-2], [111]). The first term is the damping-like torque expected in any $\mathrm{HM} / \mathrm{FM}$ heterostructures lacking inversion symmetry. The second term ( $3 \mathrm{~m}$ torque) is specific to the $3 m 1$ point group. In Supplementary Section 9, we found that the magnetization reversal of the field-free switching in $\mathrm{CuPt} / \mathrm{CoPt}$ is dominated by the nucleation of small magnetic domains. To understand how the damping-like and the $3 \mathrm{~m}$ torques cooperate and lead to deterministic switching of small magnetic domains, we consider a ferromagnetic macrospin with uniaxial perpendicular anisotropy, subjected to these two torque components. The magnetic energy reads ${ }^{17}$ (see Supplementary Section 10)

$$
W=K_{z} \sin ^{2} \theta+\frac{\chi_{3 m} M_{s}}{2} \sin \left(2 \varphi+\varphi_{E}\right) \sin ^{2} \theta,(3)
$$

where $(\theta, \varphi)$ are the polar and azimuthal angles of the magnetization direction in the $(x, y, z)$ 
frame. Depending on the direction and sign of the current, the second term either competes or cooperates with the perpendicular anisotropy. Setting $\chi_{3 m}=\eta \chi_{D L}$ and using a Gaussian pulse, $\chi_{D L}=\chi_{D L}^{0} e^{-\left(\frac{t-t_{0}}{\tau}\right)^{2}}$, we compute the final state of this macrospin (see Supplementary Fig. 22-24). Fig. 4a shows the calculated stability diagram using this model. This figure shows that for $\varphi_{E}=0^{\circ}, 60^{\circ}$, and $120^{\circ}$, reversible switching is obtained. Noticeably, the hysteresis obtained for $\varphi_{E}=0^{\circ}$ and $\varphi_{E}=120^{\circ}$ have the same polarity, while it is opposite for $\varphi_{E}=60^{\circ}$. Finally, along $\varphi_{E}=30^{\circ}, 90^{\circ}$, and $150^{\circ}$, no reversible switching is obtained (see Supplementary Fig. 23). This stability diagram is consistent with the experimental data in Fig. 2e. In order to further clarify the mechanism, we propose a scenario for the field-free switching. The scenario is depicted in Fig. 4b-f. At the initial state, the magnetization is aligned along $-\boldsymbol{z}$ (Fig. 4b) and the only torque that is active is the damping-like torque

$$
\boldsymbol{T} \sim \chi_{D L} \boldsymbol{m} \times[(\mathbf{z} \times \boldsymbol{E}) \times \boldsymbol{m}] \equiv \mathbf{z} \times \boldsymbol{E} .(4)
$$

The $3 \mathrm{~m}$ torque vanishes because $m_{x}, m_{y}=0$. Under the damping-like torque, the magnetization is brought close to the plane, along the direction perpendicular to the electric field, $\boldsymbol{m} \| \mathbf{z} \times \boldsymbol{E}$. In this case, the damping-like torque vanishes by definition, and only the $3 \mathrm{~m}$ torque is active, so

$$
\boldsymbol{T} \sim \chi_{3 m} \boldsymbol{m} \times\left[\left(m_{y} E_{x}+m_{x} E_{y}\right) \boldsymbol{x}+\left(m_{x} E_{x}-m_{y} E_{y}\right) \boldsymbol{y}\right]
$$

Now the influence of this torque on the final magnetization state depends on the direction of the electric field, as displayed in Fig. 4c-e. If the electric field is applied along $\varphi_{E}=0^{\circ}$. $\boldsymbol{E}=\boldsymbol{E} \boldsymbol{x}($ Fig. 4c), the magnetization lies along $\boldsymbol{m} \| \boldsymbol{y}$, and the $3 \mathrm{~m}$ torque reads

$$
\boldsymbol{T} \sim \chi_{3 m} \boldsymbol{m} \times \boldsymbol{E} \equiv-\chi_{3 m} \boldsymbol{z},(6)
$$

and pushes the magnetization towards $+\boldsymbol{z}$ (based on the experimental results, $\chi_{3 m}<0$ ). In other words, if the magnetization was initially along $-z$, the $3 \mathrm{~m}$ torque favors magnetization reversal. If the electric field is applied along $\varphi_{E}=30^{\circ}, \boldsymbol{E}=E\left(\frac{\sqrt{3}}{2} \boldsymbol{x}+\frac{1}{2} \boldsymbol{y}\right)$, see Fig. 4 d, the 
magnetization lies along $\boldsymbol{m} \|\left(-\frac{1}{2} \boldsymbol{x}+\frac{\sqrt{3}}{2} \boldsymbol{y}\right)$ and the $3 \mathrm{~m}$ torque vanishes $\boldsymbol{T} \sim 0$. In other words, there is no preferential direction and no reversible switching occurs. Finally, if the electric field is applied along $\varphi_{E}=60^{\circ}, \boldsymbol{E}=E\left(\frac{1}{2} \boldsymbol{x}+\frac{\sqrt{3}}{2} \boldsymbol{y}\right)$, see Fig. 4e, the magnetization lies along $\boldsymbol{m} \|\left(-\frac{\sqrt{3}}{2} \boldsymbol{x}+\frac{1}{2} \boldsymbol{y}\right)$ and the $3 \mathrm{~m}$ torque reads

$$
\boldsymbol{T} \sim-\chi_{3 m} \boldsymbol{m} \times \boldsymbol{E} \equiv \chi_{3 m} \boldsymbol{z},(7)
$$

and pushes the magnetization towards $\boldsymbol{- z}$. As a result, reversible magnetization reversal is favored but the hysteresis has an opposite sign compared to the case $\varphi_{E}=0^{\circ}$. The above scenario well interprets the symmetry-dependent field-free switching in the $L 1_{1} \mathrm{CuPt} / \mathrm{CoPt}$ system, and it may also explain the threshold current for the $\Delta H_{\mathrm{OOP}}$ in Fig. $3 \mathrm{~h}$ since the $3 \mathrm{~m}$ torque can only act on the magnetization when the injected current is large enough to bring the magnetization close to the plane.

As the $3 \mathrm{~m}$ torque originates from a peculiar symmetry (3-fold rotation and mirror symmetry) of the CuPt crystal, its strength is governed not only by the spin-orbit coupling but also the bulk crystal field. Therefore, engineering the chemical bonds within the material to enhance the crystal field could increase the torque magnitude. This observation calls for further materials exploration. In our experiments, the strength of $\Delta H_{\mathrm{OOP}}$ in $L 1_{1} \mathrm{CuPt}(13$ $\mathrm{nm}) / \mathrm{CoPt}(4 \mathrm{~nm})$ bilayer shows a strong dependence on the deposition temperature of $\mathrm{CuPt}$ $\left(T_{\mathrm{CuPt}}\right)$. As $T_{\mathrm{CuPt}}$ decreases from $500{ }^{\circ} \mathrm{C}$ to $200{ }^{\circ} \mathrm{C}, \Delta H_{\mathrm{OOP}} / J_{\text {pulse }}$ decreases more than half (Supplementary Section 11 and 12). We also replaced the ferromagnetic layer in $\mathrm{CuPt} / \mathrm{CoPt}$, by preparing two $\mathrm{CuPt}(10 \mathrm{~nm}) /[\mathrm{Co} / \mathrm{Ni}]_{5}(4 \mathrm{~nm})$ bilayers, where one has a $\mathrm{CuPt}$ layer deposited at $500{ }^{\circ} \mathrm{C}$ and the other has a $\mathrm{CuPt}$ layer deposited at room temperature. We found that the $500{ }^{\circ} \mathrm{C}-\mathrm{CuPt} /[\mathrm{Co} / \mathrm{Ni}]_{5}$ bilayer exhibited symmetry-dependent field-free switching, while the room temperature- $\mathrm{CuPt} /[\mathrm{Co} / \mathrm{Ni}]_{5}$ bilayer didn't show an observable field-free switching (Supplementary Section 13). These above experimental results imply that the good 
crystallinity and the good chemical ordering of the CuPt layer are prerequisites for the generation of the $3 \mathrm{~m}$ torque. We note that the field-free switching in Fig. 2 is incomplete if we compare $\Delta R_{\mathrm{I}}$ (Fig. 2e) with the anomalous Hall loop (Fig. 2c). This could be due to the pinning effect from the Hall voltage arms. Therefore, we fabricated the CoPt layer on CuPt into a round pillar (Fig. 5a). Fig. 5b and Fig. 5c show the anomalous Hall loop and the current-induced switching loop of the pillar device for $\theta_{\mathrm{I}}=0^{\circ}$, where we observed a complete switching. For further verification, we used the magneto-optical Kerr effect (MOKE) microscopy to check the magnetization states before and after SOT switching (see Supplementary Section 14). As shown in Fig. 5d, we firstly applied a saturation magnetic field along $-\boldsymbol{z}$ direction to initialize the magnetization of the pillar to $-\boldsymbol{M}_{\boldsymbol{z}}$. Then we removed the external magnetic field and applied a $+22 \mathrm{~mA}$ pulsed current into the channel, and we found the pillar area turned black, representing a $+\boldsymbol{M}_{z}$ state (Fig. 5e). Next, by successively applying $-22 \mathrm{~mA}$ and $+22 \mathrm{~mA}$ pulse, the color in the pillar area become grey $\left(-\boldsymbol{M}_{\boldsymbol{z}}\right.$, Fig. $\left.5 \mathrm{f}\right)$ and black ( $+\boldsymbol{M}_{z}$, Fig. $\left.5 \mathrm{~g}\right)$, respectively. Comparing the colors in Fig. $5 \mathrm{f}$ with Fig. 5d, we found that a $-22 \mathrm{~mA}$ pulse can fully recover the saturated magnetization $\left(-\boldsymbol{M}_{\boldsymbol{z}}\right)$. On the other side, we also confirmed a $+22 \mathrm{~mA}$ pulse can fully recover the $+\boldsymbol{M}_{z}$ state by comparing Fig. $5 \mathrm{j}$ with Fig. 5h. The above MOKE measurement further confirms the full SOT switching in $\mathrm{CuPt} / \mathrm{CoPt}$. In summary, our work demonstrates the observation of the out-of-plane spin-torque in the $L 1_{1} \mathrm{CuPt} / \mathrm{CoPt}$ bilayer due to the low-symmetry property at the interface. Since there are different kinds of low-symmetry point groups at various SOT interfaces, it is expected that corresponding "low-symmetry" spin-torques could exist. Compared with previous field-free switching of perpendicular switching with extra functional layers or additional asymmetry designs, the field-free switching in $L 1_{1} \mathrm{CuPt} / \mathrm{CoPt}$ is simpler in design, and possess good thermal stability and good endurance, which makes it a promising candidate for magnetic memories. 


\section{References}

1. Miron, I. M. et al. Perpendicular switching of a single ferromagnetic layer induced by in-plane current injection. Nature 476, 189-193 (2011).

2. Liu, L. et al. Spin-torque switching with the giant spin Hall effect of tantalum. Science 336, 555-558 (2012).

3. Manchon, A. et al. Current-induced spin-orbit torques in ferromagnetic and antiferromagnetic systems. Rev. Mod. Phys. 91, 035004 (2019).

4. Liu, L., Lee, O. J., Gudmundsen, T. J., Ralph, D. C. \& Buhrman, R. A. Currentinduced switching of perpendicularly magnetized magnetic layers using spin torque from the spin Hall effect. Phys. Rev. Lett. 109, 096602 (2012).

5. Pai, C. F. et al. Spin transfer torque devices utilizing the giant spin Hall effect of tungsten. Appl. Phys. Lett. 101, 122404 (2012).

6. Lau, Y. C., Betto, D., Rode, K., Coey, J. M. D. \& Stamenov, P. Spin-orbit torque switching without an external field using interlayer exchange coupling. Nat. Nanotechnol. 11, 758-762 (2016).

7. Oh, Y. W. et al. Field-free switching of perpendicular magnetization through spinorbit torque in antiferromagnet/ferromagnet/oxide structures. Nat. Nanotechnol. 11, 878-884 (2016).

8. Fukami, S., Zhang, C., DuttaGupta, S., Kurenkov \& A., Ohno, H. Magnetization switching by spin-orbit torque in an antiferromagnet-ferromagnet bilayer system. Nat. Mater. 15, 535-541 (2016).

9. van den Brink, A., et al. Field-free magnetization reversal by spin-Hall effect and 
exchange bias. Nat. Commun. 7, 10854 (2016).

10. Yu, G. et al. Switching of perpendicular magnetization by spin-orbit torques in the absence of external magnetic fields. Nat. Nanotechnol. 9, 548 (2014).

11. You, L. et al. Switching of perpendicularly polarized nanomagnets with spin orbit torque without an external magnetic field by engineering a tilted anisotropy. Proc. Natl. Acad. Sci. USA 112, 10310 (2015).

12. Liu, L. et al. Current-induced magnetization switching in all-oxide heterostructures. Nat. Nanotechnol. 14, 939-944 (2019).

13. Safeer, C. K. et al. Spin-orbit torque magnetization switching controlled by geometry. Nat. Nanotechnol. 11, 143-146 (2016).

14. Kong, W. J. et al. Spin-orbit torque switching in a T-type magnetic configuration with current orthogonal to easy axes. Nat. Commun. 10, 233 (2019).

15. Baek, Sh. C. et al. Spin currents and spin-orbit torques in ferromagnetic trilayers. Nat. Mater. 17, 509-513 (2018).

16. Laref, S., Kim, K. W. \& Manchon, A. Elusive Dzyaloshinskii-Moriya interaction in monolayer $\mathrm{Fe}_{3} \mathrm{GeTe}_{2}$. Phys. Rev. B 102, 060402 (2020).

17. Johansen, Ø., Risinggård, V., Sudbø, A., Linder, J. \& Brataas, A. Current control of magnetism in two-dimensional $\mathrm{Fe}_{3} \mathrm{GeTe}_{2}$. Phys. Rev. Lett. 122, 217203 (2019).

18. Iwata, S., Yamashita, S. \& Tsunashima, S.. Perpendicular magnetic anisotropy and magneto-optical Kerr spectra of MBE-grown PtCo alloy films. IEEE Trans. Magn. 33, 3670-3672 (1997).

19. Dannenberg, A., Gruner, M. E., Hucht, A. \& Entel, P. Surface energies of stoichiometric FePt and CoPt alloys and their implications for nanoparticle 
morphologies. Phys. Rev. B 80, 245438 (2009).

20. MacNeill, D. et al. Control of spin-orbit torques through crystal symmetry in WTe2/ferromagnet bilayers. Nat. Phys. 13, 300-305 (2017).

21. Suzuki, D., Ohtake, M., Kirino, F. \& Futamoto, M. Preparation of CoPt-alloy thin films with perpendicular magnetic anisotropy on $\mathrm{MgO}(111), \mathrm{SrTiO}_{3}(111)$, and $\mathrm{Al}_{2} \mathrm{O}_{3}(0001)$ single-crystal substrates. IEEE Trans. Magn. 48, 3195-3198 (2012).

22. Garello, K. et al. Symmetry and magnitude of spin-orbit torques in ferromagnetic heterostructures. Nat. Nanotechnol. 8, 587 (2013).

23. Hayashi, M., Kim, J., Yamanouchi, M. \& Ohno, H. Quantitative characterization of the spin-orbit torque using harmonic Hall voltage measurements. Phys. Rev. B 89, 144425 (2014).

24. Pi, U. H. et al. Tilting of the spin orientation induced by Rashba effect in ferromagnetic metal layer. Appl. Phys. Lett. 97, 162507 (2010).

25. Kim, J. et al. Layer thickness dependence of the current-induced effective field vector in $\mathrm{Ta}|\mathrm{CoFeB}| \mathrm{MgO}$. Nat. Mater. 12, 240-245 (2013).

26. Guillemard, C., Petit-Watelot, S., Andrieu, S. \& Rojas-Sánchez, J. C. Charge-spin current conversion in high quality epitaxial Fe/Pt systems: Isotropic spin Hall angle along different in-plane crystalline directions. Appl. Phys. Lett. 113, 262404 (2018).

27. Pai, C. F., Mann, M., Tan, A. J. \& Beach, G. S. D. Determination of spin torque efficiencies in heterostructures with perpendicular magnetic anisotropy. Phys. Rev. B 93, 144409 (2016).

28. Železný, J. et al. Spin-orbit torques in locally and globally noncentrosymmetric crystals: Antiferromagnets and ferromagnets. Phys. Rev. B 95, 014403 (2017).

\section{Acknowledgements}


The research is supported by the Singapore National Research Foundation under CRP Award No. NRF-CRP10-2012-02 and the Singapore Ministry of Education MOE2018-T2-1-019, MOE2018-T2-2-043, A*STAR Grant No. A1983C0036, A*STAR IAF-ICP 11801E0036, MOE Tier1 R-284-000-195-114 and the King Abdullah University of Science and Technology (KAUST). J.S.C. is a member of the Singapore Spintronics Consortium (SGSPIN).

\section{Author contributions}

L.L. and J.S.C.: conceived and designed the experiments. L.L. and C.Z.: performed thin film deposition, device fabrication, transport measurements, and data analysis. A.M.: theoretically proposed the $3 \mathrm{~m}$ torque and performed the calculation. T.Z., X.S., J.D., Q.X., S.C., S.S., and J.Y.: contributed to thin film deposition and device fabrication. W.L., J.Z., R.G., H.W., and P.Y.: contributed to data analysis. C.L. and S.P.: performed STEM experiments. L.L., C.Z., A.M., and J.S.C.: wrote the manuscript and all authors contributed to its final version.

\section{Competing financial interests's statement}

The authors declare no competing financial interests.

Correspondence and requests for materials should be addressed to J.S.C. (msecj@nus.edu.sg) and A.M. (manchon@cinam.univ-mrs.fr)

\section{Data availability}

The authors declare that the main data supporting the findings of this study are available within the Letter and its Supplementary Information. Extra data are available from the corresponding author upon reasonable request. Soure Data are provided with this paper. 

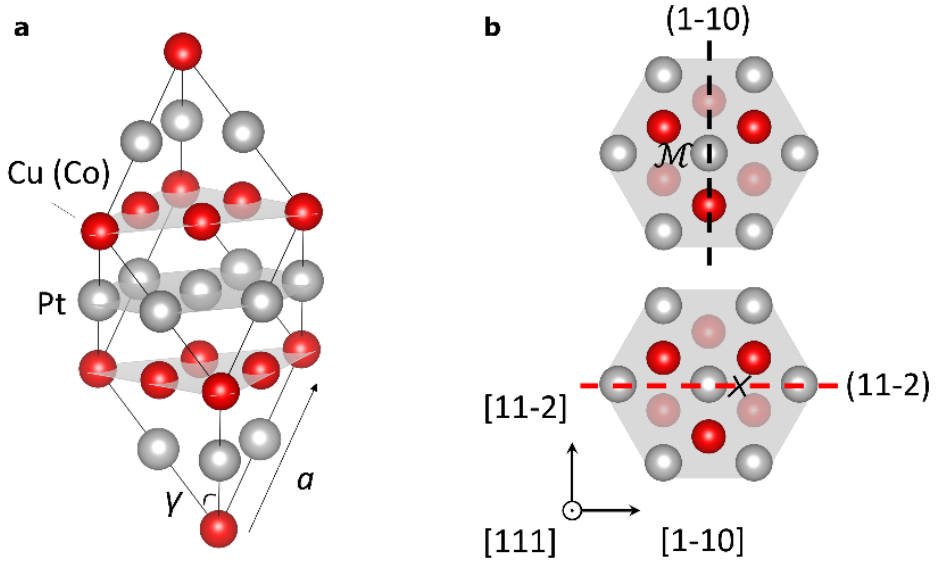

c

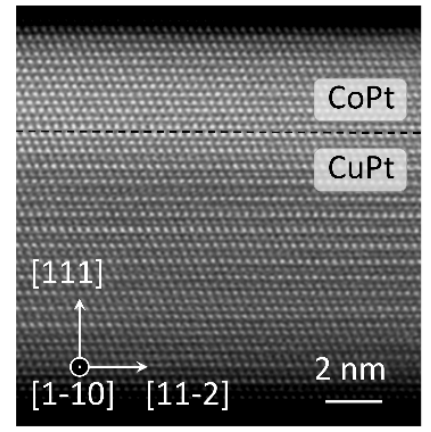

Figure 1| Crystal structure and symmetry analysis. a, The crystal structure of $L 1_{1} \mathrm{CuPt}$ $(\mathrm{CoPt})$. The lattice parameter $a$ is $5.340 \AA$ and the lattice angle $\gamma$ is $61.5^{\circ} . \mathbf{b}$, Plane view of the $L 1_{1}$ hexagon projected along the [111] direction where the Pt atomic layer (grey color) was sandwiched by two $\mathrm{Cu}(\mathrm{Co})$ atomic layers (heavy red on top and light red on bottom). For simplicity, only the atoms within the hexagon region (grey colored) are shown. The mirror symmetry " $\mathcal{M}$ " is preserved in the (1-10) plane (dark dashed line), while broken in the (11-2) plane (red dashed line). c, High-angle annular dark-field-STEM images of the CoPt (4 $\mathrm{nm}) / \mathrm{CuPt}(10 \mathrm{~nm})$ bilayer. The dashed line indicated the $\mathrm{CuPt} / \mathrm{CoPt}$ interface.
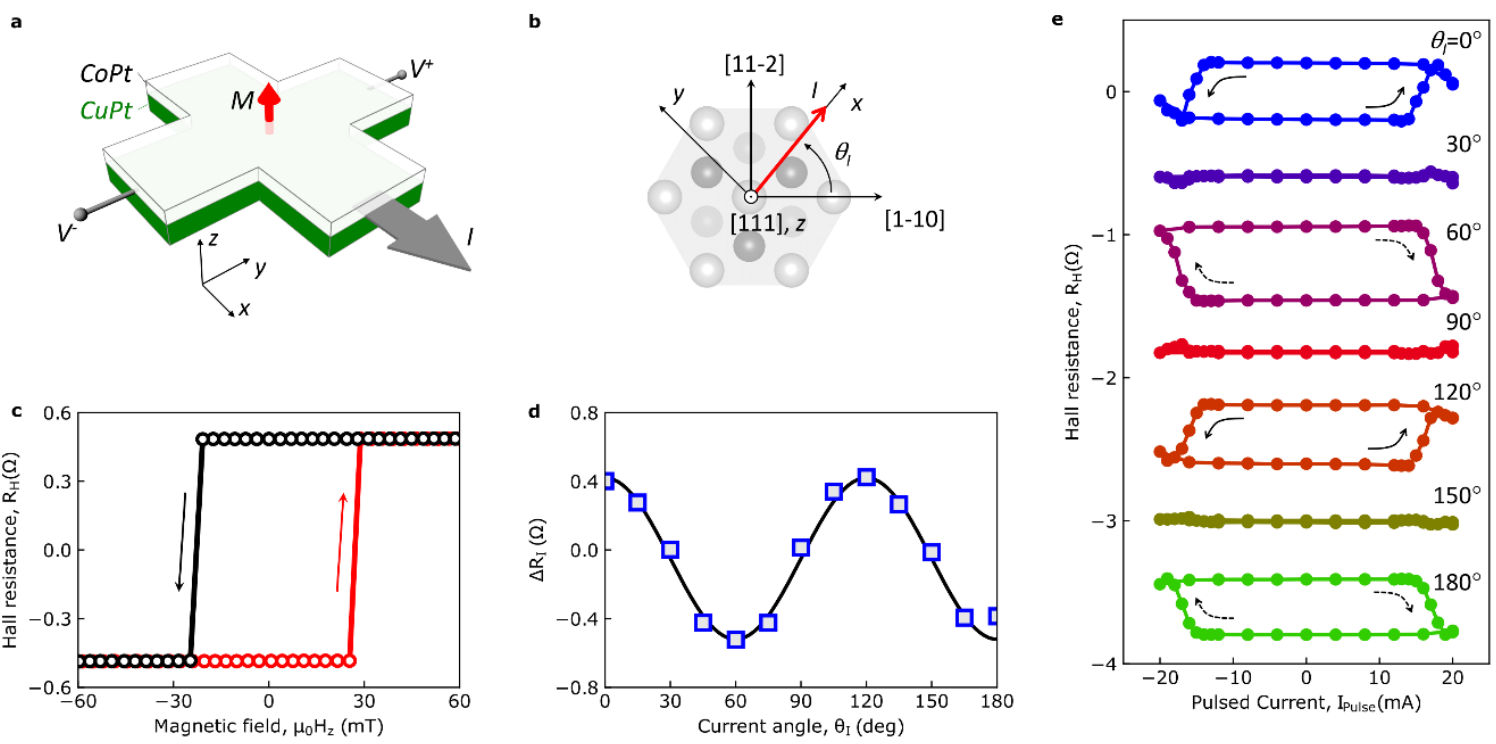


\section{Figure 2| Symmetry-dependent magnetic field-free magnetization switching. a,}

Schematic of the $\mathrm{CuPt} / \mathrm{CoPt}$ Hall bar for electrical transport measurement. The red arrow represents the magnetization $(M)$. The grey arrow represents the current $(I)$ flowing. The $x$ and $y$-axis are the same as that in $(\mathbf{b})$. $\mathbf{b}$, The definition of current flowing direction $\left(\theta_{\mathrm{I}}\right)$. The current is applied along the Hall bar, which has an azimuth angle of $\theta_{I}$ with respect to the [110] direction. c, Anomalous Hall effect of the bilayer for $\theta_{I}=0^{\circ}$. d, Current angle dependence of the SOT induced magnetization switching. The solid line is a cosine fit to the data. e, Current-induced magnetization switching for Hall bars with different $\theta_{\mathrm{I}}$. The dashed (solid) arrows indicate clockwise (anti-clockwise) switching polarity. The loops were manually shifted for better visualization. The pulse width is $30 \mu$ s.
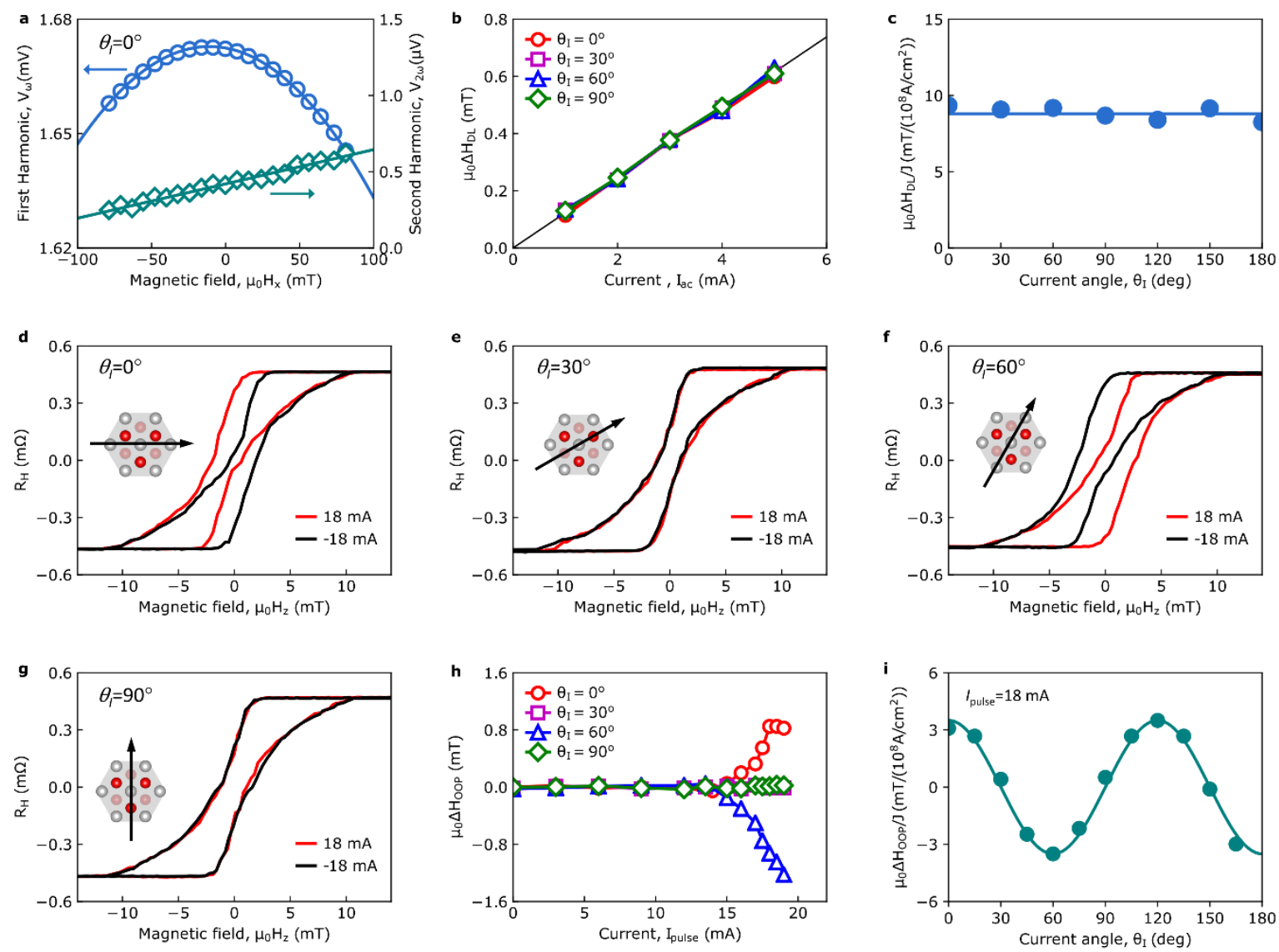

Figure 3| Symmetry dependence of current-induced effective fields. a, First and second harmonic Hall signals as a function of the in-plane magnetic field $\left(H_{x}\right)$ under $5 \mathrm{~mA}$ 
(amplitude) a.c. excitation current. b, Current dependence of damping-like effective field for $\theta_{I}=0^{\circ}$ (red), $\theta_{I}=30^{\circ}$ (purple), $\theta_{I}=60^{\circ}$ (blue) and $\theta_{I}=90^{\circ}$ (green). c, Damping-like effective fields obtained for varying $\theta_{I}$. The solid line is a guide to the eye. d-g, Anomalous Hall loops under $+18 \mathrm{~mA}$ and $-18 \mathrm{~mA}$ pulsed d.c. current for $\theta_{I}=0^{\circ}, \theta_{I}=30^{\circ}, \theta_{I}=60^{\circ}$, and $\theta_{I}=90^{\circ}$. The inset in each figure indicates the current direction. $\mathbf{h}$, Current dependence of out-of-plane effective field for $\theta_{I}=0^{\circ}$ (red), $\theta_{I}=30^{\circ}$ (purple), $\theta_{I}=60^{\circ}$ (blue) and $\theta_{I}=90^{\circ}$ (green). The pulse width is $30 \mu \mathrm{s}$. i, Out-of-plane effective field as a function of $\theta_{I}$. The solid line is a cosine fit to the data.
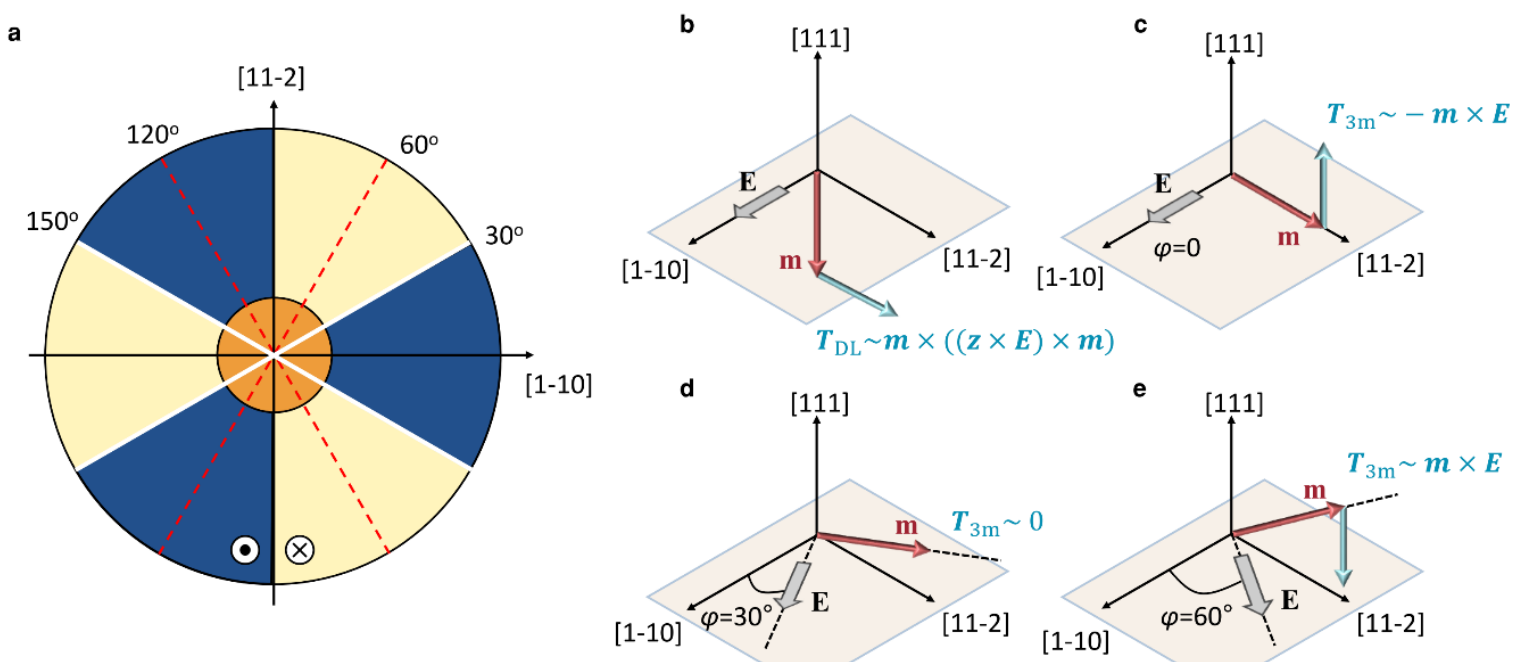

Figure 4| Theoretical model for current-induced spin-torque in $\mathrm{CuPt} / \mathrm{CoPt}$ bilayer. a,

Calculated stability diagram of the ferromagnet submitted to both damping-like and $3 \mathrm{~m}$ torques in the absence of external magnetic field. The diagram is plotted as a function of the current direction. Blue and white regions refer to $\left(m_{z}^{+}+m_{z}^{-}\right)= \pm 1$, while the central orange region refers to the bistable state $\left(m_{z}^{+}+m_{z}^{-}\right)=0$. In $m_{z}^{+}$the superscript refers to the initial magnetic configuration, up $(+)$ or down $(-)$. The white (red) lines denote directions where the $3 m$ torque vanishes (is maximum). b-f, Schematics of the field-free switching scenario described in the main text. $\mathbf{b}$, In the initial configuration, the magnetization points along $-\mathbf{z}$ 
and only experiences the damping-like torque. Once brought in the plane, the magnetization only experiences the $3 \mathrm{~m}$ torque, whose magnitude and direction depends on the direction of current injection: (c) for $\varphi_{E}=0^{\circ}$, the $3 \mathrm{~m}$ torque favors magnetization alignment along $+\mathbf{z}$, (d) for $\varphi_{E}=30^{\circ}$, the $3 \mathrm{~m}$ torque vanishes, and (e) for $\varphi_{E}=60^{\circ}$, the $3 \mathrm{~m}$ torque favors magnetization alignment along -z.
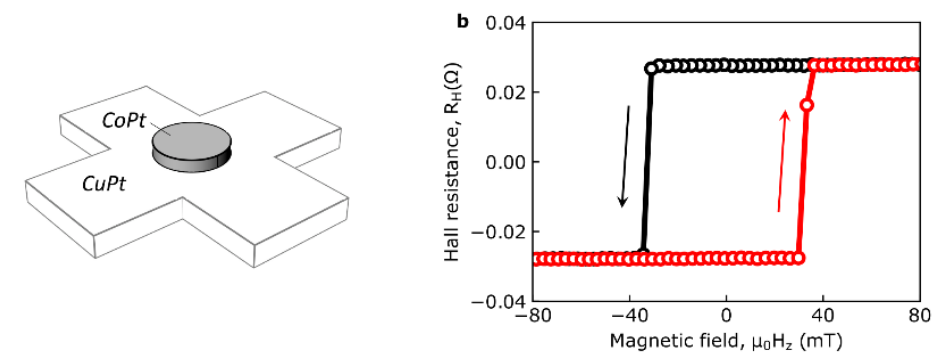
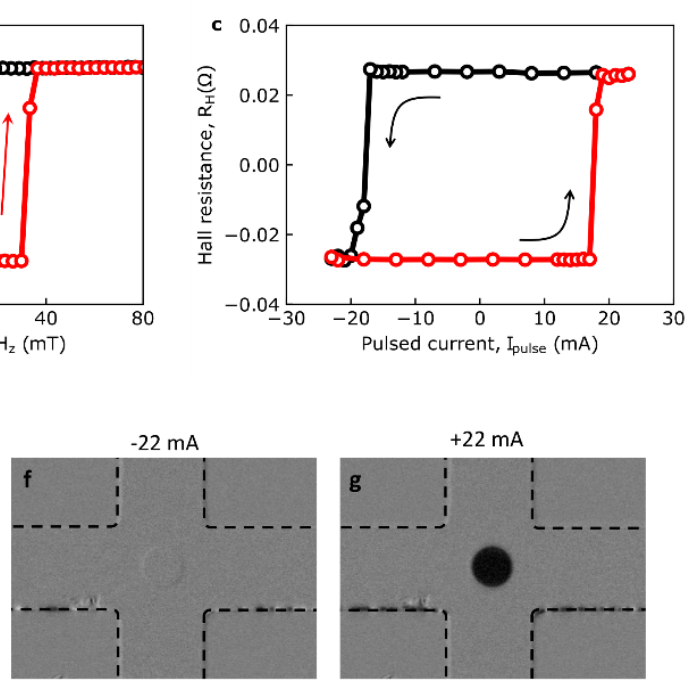

$+22 \mathrm{~mA}$
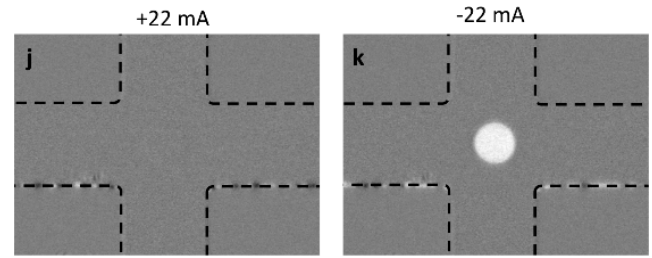

Figure 5| Current-induced field-free magnetization switching in $\mathrm{CuPt}(13 \mathrm{~nm}) / \mathrm{CoPt}(4$

nm) pillar sample. a, Schematic of the $\mathrm{CuPt} / \mathrm{CoPt}$ pillar device for switching measurement.

b, Anomalous Hall effect of the pillar device with $\theta_{I}=0^{\circ}$. c, Current-induced magnetization switching of the pillar device with $\theta_{I}=0^{\circ} . \mathbf{d}-\mathbf{k}$, MOKE imaging of the CoPt pillar during field-free SOT switching. The black dashed lines in the figures outline the Hall bar edges. The red lines in (a) and (e) outline the pillar edges. For processes (d-g), we firstly applied a negative magnetic field along the $-\boldsymbol{z}$ direction to initialize the magnetization to $-\boldsymbol{M}_{\boldsymbol{z}}$. To enhance the contrast, the background in the whole image was subtracted such that the colors 
within and outside the pillar region are the same (grey). However, only the grey color in the pillar area represents a $-\boldsymbol{M}_{z}$ state $(\mathbf{d})$, since there is no CoPt outside the pillar region. After that, we successively applied $+22 \mathrm{~mA}(\mathbf{e}),-22 \mathrm{~mA}(\mathbf{f})$, and $+22 \mathrm{~mA}(\mathbf{g})$ into the CuPt channel to check the magnetization evolution. The dark color in (e) and (g) represent a $+\boldsymbol{M}_{z}$ state. For processes (h-k), we firstly applied a positive magnetic field along the $+\boldsymbol{z}$ direction to initialize the magnetization to $+\boldsymbol{M}_{z}$. Then the whole background was subtracted and the grey color in the pillar area represents $+\boldsymbol{M}_{\boldsymbol{z}}(\mathbf{h})$. After that, we successively applied $-22 \mathrm{~mA}(\mathbf{i}),+22 \mathrm{~mA}(\mathbf{j})$, and $-22 \mathrm{~mA}(\mathbf{k})$ into the CuPt channel to check the magnetization evolution. The white color in (i) and (k) represents a $-\boldsymbol{M}_{\boldsymbol{z}}$ state. The pulse width of the current for the measurement is 30 $\mu \mathrm{s}$.

\section{Methods}

Sample growth and device fabrication. $\mathrm{CuPt}(10 \mathrm{~nm}) / \mathrm{CoPt}(4 \mathrm{~nm})$ bilayer was epitaxially deposited on $\mathrm{SrTiO}_{3}$ (111) single-crystal substrate by d.c. magnetron sputtering (AJA). For the deposition of $\mathrm{CuPt}$, the Ar pressure is $5 \mathrm{mTorr}$ and the temperature is $500{ }^{\circ} \mathrm{C}$. The sputtering powers for $\mathrm{Cu}$ target and $\mathrm{Pt}$ target are both $50 \mathrm{~W}$. For the deposition of $\mathrm{CoPt}$, the Ar pressure is 6 mTorr and the temperature is $300{ }^{\circ} \mathrm{C}$. The sputtering powers for Co target and Pt target are $32 \mathrm{~W}$ and $21 \mathrm{~W}$, respectively. The nominal deposition rates for $\mathrm{CuPt}$ and CoPt are $0.14 \mathrm{~nm} / \mathrm{s}$ and $0.051 \mathrm{~nm} / \mathrm{s}$, respectively. Then the bilayer was left to cool down to room temperature and a $2 \mathrm{~nm} \mathrm{SiO}_{2}$ protection layer was deposited. After deposition, the films were patterned into $5 \mu \mathrm{m}$ Hall bars with different in-plane orientations by using laser writer and Ar ion milling. Then the contact electrode pattern was defined by laser writer and followed by the deposition of $\mathrm{Ti}(5 \mathrm{~nm}) / \mathrm{Cu}(100 \mathrm{~nm})$ and the lift-off process. For varying $\mathrm{CuPt}$ deposition temperature measurements, $\mathrm{CuPt}(13 \mathrm{~nm}) / \mathrm{CoPt}(4 \mathrm{~nm})$ samples with $\mathrm{CuPt}$ deposited at $200{ }^{\circ} \mathrm{C}, 300{ }^{\circ} \mathrm{C}, 400{ }^{\circ} \mathrm{C}$, and $500{ }^{\circ} \mathrm{C}$ are prepared. For the $\mathrm{CuPt}(10 \mathrm{~nm}) /[\mathrm{Co} / \mathrm{Ni}]_{5}$ 
$(4 \mathrm{~nm})$, the growth conditions of the $\mathrm{CuPt}$ are the same as that for the $\mathrm{CuPt}(10 \mathrm{~nm}) / \mathrm{CoPt}(4$ $\mathrm{nm})$ sample, expect that the $\mathrm{CuPt}$ for room temperature-CuPt $(10 \mathrm{~nm}) /[\mathrm{Co} / \mathrm{Ni}]_{5}(4 \mathrm{~nm})$ sample is deposited at room temperature. The $[\mathrm{Co} / \mathrm{Ni}]_{5}$ layer is deposited at room temperature.

Current-induced switching measurement. In current-induced magnetization switching measurement, for each data point, a pulsed d.c. electrical current with a duration of $30 \mu \mathrm{s}$ was applied. After 8 seconds, the Hall resistance was recorded by using a small a.c. excitation current $(50 \mu \mathrm{A})$.

STEM sample preparation and characterization. $\mathrm{CuPt} / \mathrm{CoPt}$ STEM sample was fabricated by a focused ion beam machine (FEI Versa 3D system). The sample was thinned down using a $\mathrm{Ga}$ ion beam first with an accelerating voltage of $30 \mathrm{kV}$ and then with an accelerating voltage of $8 \mathrm{kV}$. After that, the sample was polished by a $2 \mathrm{kV}$ ion beam to minimize the beam damaged region. The structure characterization was conducted with a JEOL ARM200 STEM which is equipped with an ASCOR probe corrector operating at an accelerating voltage of $200 \mathrm{kV}$. The HAADF image was acquired with a probe forming angle of $30 \mathrm{mrad}$ and collection inner and outer semiangles of 68 and $280 \mathrm{mrad}$, respectively.

MOKE measurement. We use a MOKE from Evico Magnetics to image the magnetic domains during the SOT switching. In order to enhance the contrast, we subtract the background signal for the whole view after each initialization with an out-of-plane magnetic field. Then we removed the magnetic field and applied a pulsed current with a duration of 30 $\mu \mathrm{s}$. The MOKE image was then captured after a stabilization time longer than $2 \mathrm{~s}$. 